\title{
Two Indices Affecting the Directions of the Sylvian Fissure Dissection in Middle Cerebral Artery Bifurcation Aneurysms
}

\author{
Hyun Wook Park', Seung Young Chung', Moon Sun Park', Seong Min Kim', Byul Hee Yoon', Han Kyu Kim² \\ 'Department of Neurosurgery, Eulji University Hospital, College of Medicine, Eulji University, Taejon, Korea \\ ${ }^{2}$ Department of Neurosurgery, Kosin University Hospital, College of Medicine, Kosin University, Busan, Korea
}

Objective : This study proposes more objective methods for deciding the appropriate direction of the sylvian fissure dissection during surgical clipping in middle cerebral artery (MCA) bifurcation aneurysms.

Methods: We reviewed data of 36 consecutive patients with MCA bifurcation aneurysms. We measured 2 indices preoperatively on 3-dimensional computed tomography angiography (3D-CTA). Analysis of the calculated data allowed us to select the appropriate direction of sylvian fissure dissection for ease of proximal control of M1. Statistically, Mann-Whitney test was used.

Results : We classified subjects into 2 groups based on the technical level of $\mathrm{M} 1$ exposure during surgical clipping. When it was difficult to expose M1, subjects were assigned to Group I, and Group II were subjects in whom M1 exposure was easy. The mean difference between the distances extending from the limbus sphenoidale (LS) line to the internal carotid artery bifurcation and extending from the LS line to the MCA bifurcation was $1.00 \pm 0.42 \mathrm{~mm}$ in group I and $4.39 \pm 2.14 \mathrm{~mm}$ in group II. The mean $\mathrm{M} 1$ angle was $9.36 \pm 3.73^{\circ}$ in the group I and $34.05 \pm$ $16.71^{\circ}$ in the group II (M1 slope gap $p<0.05, \mathrm{M} 1$ angle $p<0.05$ ).

Conclusion: We have found an objective method for preoperatively verifying ease of exposure of M1 artery during surgical clipping. Therefore, we suggest use of the preoperative $M 1$ slope gap and $M 1$ angle as indicators in 3D-CTA selecting the direction of sylvian fissure dissection for easy proximal control of $\mathrm{Ml}$.

Keywords Middle cerebral artery, Intracranial aneurysm, Dissecting, Three-dimensional cerebral angiography

\author{
J Cerebrovasc Endovasc Neurosurg. \\ 2013 September;15(3):164-170 \\ Received : 5 July 2013 \\ Revised : 5 August 2013 \\ Accepted : 8 August 2013
}

\begin{abstract}
Correspondence to Seung Young Chung Department of Neurosurgery, Eulji University Hospital, College of Medicine, Eulji University, 1306 Dunsan-dong, Seo-gu, Daejeon 302-799, Korea

Tel : $82-42-611-3442$

Fax : 82-42-611-3444

E-mail : neurocsy@eulji.ac.kr
\end{abstract}

This is an Open Access article distributed under the terms of the Creative Commons Attribution NonCommercial License (http://creativecommons.org/licenses/by-nc/3.0) which permits unrestricted noncommercial use, distribution, and reproduction in any medium, provided the original work is properly cited.

\section{INTRODUCTION}

There are 3 basic surgical techniques for the dissection and clipping of middle cerebral artery (MCA) bifurcation aneurysms. First, the sylvian fissure can be opened from proximal to distal; second, from distal to proximal; and third, it can be opened through an incision of the superior temporal gyrus following a sub- pial resection depending on the presence of intra-parenchymal hematoma, the size and the projection of aneurysm and the length of M1. ${ }^{89)}$ The trans-sylvian approach can efficiently expose the MCA lesions. During clipping surgery in MCA bifurcation aneurysms, the sylvian fissure can be opened proximally, followed by surgical approach from the carotid cistern to superficial sylvian cistern direction until the aneurysm is 


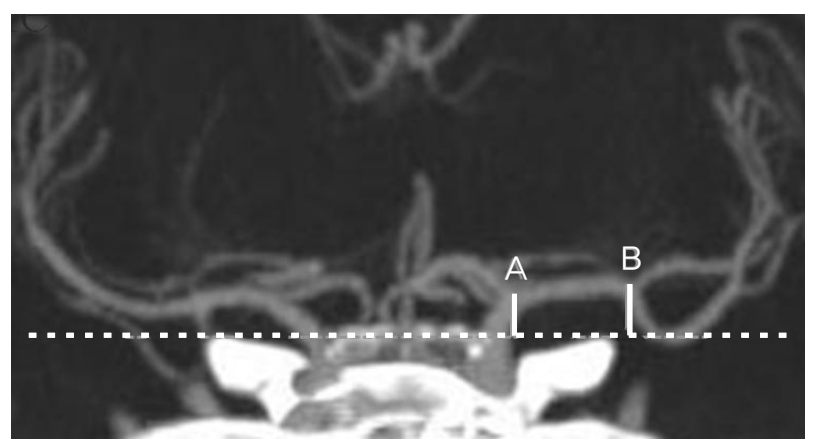

Fig. 1. The distance extending from the limbus sphenoidale line (LS; dotted line) to the internal carotid artery (ICA) bifurcation $(A)$ and that extending from the limbus sphenoidale line to middle cerebral artery (MCA) bifurcation (B).

reached. Alternatively, the sylvian fissure can also be opened distally, followed by dissection in the retrograde direction starting from the identification of the distal MCA branches such as M4 branches to main stem of the MCA arise such as M1 vessel. ${ }^{7}$ Choice of dissection methods is according to each surgeons' preference and experience. Therefore, objective indicators of surgical decision making are required, especially for inexperienced and young neurosurgeons.

Both projection of aneurysm and direction of M1 vessel are 2 important factors determining ease of exposure of M1 during surgical clipping in MCA bifurcation aneurysms. We routinely perform clipping surgery via a pterional approach, for which the head is positioned depending on the location of aneurysm. The shoulder on the operated side is elevated and the head is rotated approximately 30 degrees toward the opposite side and then extended 20 degrees. In addition, we often change the operating chair, table, head rest and microscope during the surgery to achieve an appropriate surgical field. The size, location or pathology of aneurysms are useful in describing the aneurysms, but less useful in performing a preoperative work-up such as decision on the direction of sylvian fissure dissection during clipping surgery in MCA bifurcation aneurysms. We conducted this study to propose objective methods for deciding on the appropriate direction of the sylvian fissure dissection during clipping surgery in MCA bifurcation aneurysms.

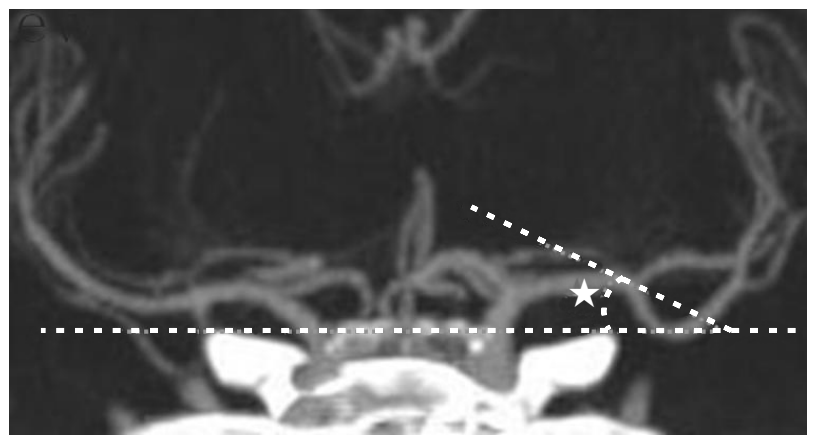

Fig. 2. M1 angle (Asterisk) between the straight segment of distal M1 at aneurysmal neck (Dotted line) and LS line.

\section{MATERIALS AND METHODS}

\section{Patient populations}

We reviewed data of 36 consecutive patients with MCA bifurcation aneurysms who underwent clipping surgery at our institute from January 2011 to January 2013. Twenty-nine of the patients are women and 7 patients are men; the mean age is 55.38 year-old (range between 33 and 77 year-old).

Among 36 patients, 21 patients had ruptured and 15 had unruptured MCA bifurcation aneurysms. In 21 patients with ruptured MCA bifurcation aneurysms, 13 patients also had intracerebral hemorrhage. All were surgically treated by clipping of aneurysm through a pterional craniotomy with distal to proximal sylvian fissure dissection. In the 13 patients with intracranial hemorrhage, hematoma removal was performed during sylvian fissure dissection.

We assigned our clinical series of patients to group I $(\mathrm{n}=17)$ or group II $(\mathrm{n}=19)$ depending on the technical level of M1 exposure by the surgeon's technical experience during surgical clipping. The technical level included minimal dissection after the aneurysm is reached, less brain retraction and the short time required to identify M1. We actually confirmed group classification in intraoperative surgical view. Group I were difficult to expose M1 exposure and Group II were ease to expose M1.

\section{Neuroimaging studies}

We used coronal planes of 3-dimensional brain com- 
puted tomography angiography (3D-CTA) \& transfemoral cerebral angiography (TFCA) as a preoperative work-up. The limbus sphenoidale (LS) is identified as a small borderline separating the planum sphenoidale and chiasmatic groove. ${ }^{5}$ In other words, the ridge at anterior border of the chiasmatic groove is the LS. We defined the LS line as an imaginary straight line that extends from the LS.

We preoperatively measured the shortest distance between the LS line and internal carotid artery (ICA) bifurcation (A) and between the LS line and MCA bifurcation (B) on coronal plane on a 3D-CTA (Fig. 1) and we calculated the value of gap (A) and (B), a so-called "M1 slope gap". We defined the "M1 angles" as an angle formed between an imaginary straight line extended from the distal M1 portion at aneurysmal neck and the LS line (Fig. 2). We predicted the traveling direction of M1 on microscopic surgical field view by measuring this M1 angles and M1 slope gap. We decided the appropriate direction of sylvian fissure dissection for ease of M1 exposure based upon our analysis of these data.

Statistical analysis was performed with commercial software (SPSS 15.0, SPSS Inc., Chicago, IL, USA). The Mann-Whitney test was used for the statistical significance of the independent variables between groups. A $p$ value of less than 0.05 was considered significant.

\section{RESULTS}

The M1 slope gap was $1.00 \pm 0.42 \mathrm{~mm}$ in the group I and $4.39 \pm 2.14 \mathrm{~mm}$ in the group II. The mean M1 angle was $9.36 \pm 3.73^{\circ}$ in the group I and $34.05 \pm$ $16.71^{\circ}$ in the group II (Table 1).

M1 slope gap and M1 angles between Group I and II differed significantly by the Mann-Whitney test, a non-parametric test (M1 slope gap $p<0.05$, M1 angle $p<0.05)$.

\section{Illustrative case}

\section{Case I}

A 48-year-old woman presented with drowsy men- tality due to a ruptured left MCA bifurcation aneurysm. It was difficult to expose the M1 vessel for proximal control through distal to proximal sylvian fissure dissection, so she is assigned to group I. The M1 slope gap was $1.31 \mathrm{~mm}$ and the M1 angles was $10.2^{\circ}$ (Fig. 3). We confirmed this in the intraoperative surgical view (Fig. 4).

\section{Case II}

A 65-year-old woman presented with severe headache due to a ruptured right MCA bifurcation aneurysm. It was easy to expose the M1 vessel for proximal control through distal to proximal sylvian fissure dissection so she is assigned to group II. The M1 slope gap was $6.53 \mathrm{~mm}$ and the M1 angles was $51.7^{\circ}$ (Fig. 5). We confirmed this in the intraoperative surgical view (Fig. 6).

\section{DISCUSSION}

MCA aneurysms account for $20-25 \%$ of all intracranial aneurysms, almost $80 \%$ of which occur at the MCA bifurcation. ${ }^{3) 4)}$ The middle cerebral artery is divided into 4 segments. The main trunk of the MCA starts at the ICA bifurcation in the sylvian cistern. The M1 segment(sphenoidal segment) runs laterally until it reaches the level of the limen insula where it splits usually into 2 branches, the superior division (upper trunk) and inferior division (lower trunk). ${ }^{6}$ ) The M2 segment (insular segment) starts from the genu portion to circular sulcus and runs parallel with the insular surface, supplying blood to the insula. The M3 segment (opercular segment) runs from the insular sulcus to the superficial part of sylvian fissure. The M4 segment (cortical segment) runs from the superficial sylvian fissure to the cerebral cortex, ending as terminal branches. ${ }^{4)}$

Sylvian fissure dissection can be performed during clipping of MCA bifurcation aneurysms. With a trans-sylvian approach, the MCA lesions can be efficiently exposed. There are 2 methods of sylvian fis- 
HYUN WOOK PARK ET AL

Table 1. Patients and angiographic data of 36 patients with MCA bifurcation aneurysms including M1 slope gap and M1 angle measurement of group I and group II on pre-operative 3D-CTA.

\begin{tabular}{|c|c|c|c|c|c|c|}
\hline Group & Sex/Age & A & $B$ & M1 slope gap & M1 angle & Note \\
\hline \multirow[t]{17}{*}{ Group I } & $F / 57$ & 6.45 & 7.42 & 0.97 & 5.80 & $\mathrm{ICH}$ \\
\hline & $\mathrm{F} / 77$ & 10.81 & 11.34 & 0.53 & 7.40 & \\
\hline & $F / 48$ & 2.41 & 3.72 & 1.31 & 10.20 & $\mathrm{ICH}$ \\
\hline & $\mathrm{F} / 71$ & 7.73 & 6.84 & 0.89 & 10.39 & $\mathrm{ICH}$ \\
\hline & $F / 68$ & 7.98 & 9.06 & 1.06 & 2.00 & \\
\hline & $F / 68$ & 2.05 & 3.15 & 1.10 & 9.10 & Unruptured \\
\hline & $F / 53$ & 1.06 & 2.13 & 1.07 & 14.00 & Unruptured \\
\hline & $M / 60$ & 8.92 & 7.92 & 1.00 & 17.00 & Unruptured \\
\hline & $M / 52$ & 5.52 & 4.04 & 1.48 & 13.10 & Unruptured \\
\hline & $\mathrm{F} / 52$ & 3.83 & 2.17 & 1.66 & 9.90 & Unruptured \\
\hline & $\mathrm{F} / 51$ & 4.52 & 4.00 & 0.52 & 9.70 & Unruptured \\
\hline & $M / 63$ & 2.93 & 1.44 & 1.49 & 7.20 & Unruptured \\
\hline & $M / 44$ & 5.94 & 5.43 & 0.51 & 5.50 & Unruptured \\
\hline & $F / 58$ & 7.98 & 6.91 & 1.07 & 9.25 & $\mathrm{ICH}, \mathrm{IVH}$ \\
\hline & $M / 44$ & 4.95 & 6.43 & 1.48 & 4.60 & \\
\hline & $\mathrm{F} / 77$ & 3.10 & 2.97 & 0.13 & 12.00 & \\
\hline & $\mathrm{F} / 51$ & 5.84 & 5.05 & 0.79 & 11.97 & \\
\hline Avg. & & $5.41( \pm 2.69)$ & $5.33( \pm 2.73)$ & $1.00( \pm 0.42)$ & $9.36( \pm 3.73)$ & \\
\hline \multirow[t]{19}{*}{ Group II } & $\mathrm{F} / 44$ & 4.30 & 7.81 & 3.51 & 2.66 & $\mathrm{ICH}$ \\
\hline & $F / 36$ & 7.82 & 10.78 & 2.96 & 3.34 & $\mathrm{ICH}$ \\
\hline & $F / 69$ & 4.45 & 10.90 & 6.45 & 32.10 & Unruptured \\
\hline & $\mathrm{F} / 53$ & 8.63 & 4.65 & 3.98 & 22.04 & Unruptured \\
\hline & $F / 33$ & 1.89 & 7.56 & 5.67 & 22.48 & $\mathrm{ICH}$ \\
\hline & $F / 39$ & 9.84 & 6.56 & 3.28 & 28.57 & $\mathrm{ICH}$ \\
\hline & $\mathrm{F} / 42$ & 1.80 & 6.30 & 4.50 & 53.00 & Unruptured \\
\hline & $\mathrm{F} / 43$ & 0.51 & 5.02 & 4.51 & 21.00 & Unruptured \\
\hline & $F / 49$ & 4.20 & 3.66 & 0.54 & 29.46 & \\
\hline & $F / 67$ & 6.82 & 10.88 & 4.06 & 30.10 & Unruptured \\
\hline & $F / 66$ & 3.14 & 1.31 & 1.83 & 36.87 & $\mathrm{ICH}, \mathrm{IVH}$ \\
\hline & $M / 47$ & 8.51 & 5.34 & 3.17 & 41.00 & $\mathrm{ICH}, \mathrm{IVH}$ \\
\hline & $M / 66$ & 9.46 & 5.48 & 3.98 & 44.00 & Unruptured \\
\hline & $F / 61$ & 10.25 & 5.75 & 4.50 & 42.95 & Unruptured \\
\hline & $\mathrm{F} / 44$ & 6.57 & 17.36 & 10.79 & 28.30 & $\mathrm{ICH}$ \\
\hline & $F / 64$ & 7.55 & 10.51 & 2.96 & 44.30 & $\mathrm{ICH}$ \\
\hline & $F / 65$ & 8.64 & 2.11 & 6.53 & 51.70 & $\mathrm{ICH}$ \\
\hline & $F / 61$ & 6.52 & 1.80 & 4.72 & 72.80 & \\
\hline & $F / 51$ & 6.17 & 0.69 & 5.48 & 40.30 & \\
\hline Avg. & & $4.94( \pm 2.91)$ & $6.55( \pm 4.17)$ & $4.39( \pm 2.14)$ & $34.05( \pm 16.71)$ & \\
\hline
\end{tabular}

A: Distance between the LS line and ICA bifurcation on coronal plane on a 3-D CTA; B: Distance between LS line and the MCA bifurcation on coronal plane on a 3-D CTA. $\mathrm{ICH}=$ intracerebral hemorrhage; $\mathrm{IVH}=$ intraventricular hemorrhage; 3-D CTA=3-dimensional computed tomography angiography; Avg = average.

sure dissections. First, the sylvian fissure can be opened from proximal to distal dissection. Second, the sylvian fissure can also be opened from distal to prox- imal dissection in the retrograde direction starting from the identification of the cortical branches of MCA to main stem of the MCA arise. In the proximal 


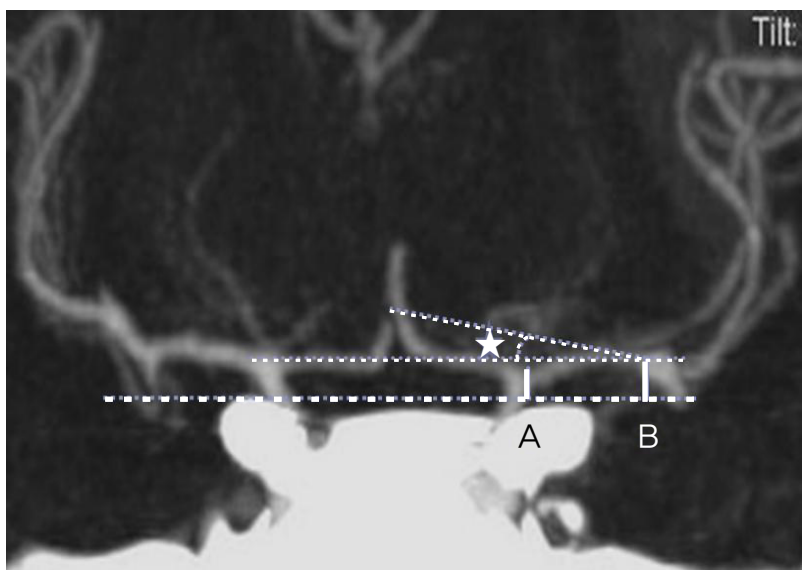

Fig. 3. The distance from the LS line to the ICA bifurcation is $2.41 \mathrm{~mm}(\mathrm{~A})$. The distance from the LS line to the MCA bifurcation is $3.72 \mathrm{~mm}(\mathrm{~B})$ and the difference between 2 lines is $1.31 \mathrm{~mm}$. The $\mathrm{M} 1$ angle is 10.2 (Asterisk).

to distal trans-sylvian approach, the carotid cistern and chiasmatic cistern are opened initially. This is followed by the removal of cerebrospinal fluid (CSF). This achieves brain relaxation. Early proximal exposure of an aneurysm is achieved by first opening the optico-carotid cistern and M1 exposure for M1 proximal control, but the frontal lobe can be damaged by early subfrontal retraction while the optico-carotid cistern is identified and opened. ${ }^{11)}$ The distal to proximal trans-sylvian approach is advantageous in that it allows a wider dissection of sylvian fissure. Also, the brain retraction can be minimized and the lenticulo-striate arteries injury prevented. ${ }^{7}$ In this ap-

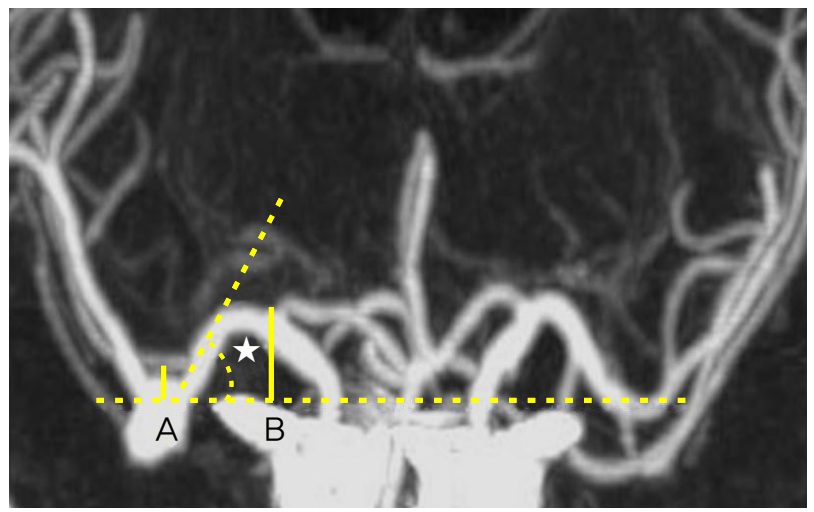

Fig. 5. The distance from the LS line to the ICA bifurcation is $8.64 \mathrm{~mm}(\mathrm{~A})$. The distance from the $\mathrm{LS}$ line to the MCA bifurcation is $2.11 \mathrm{~mm}(\mathrm{~B})$ and the difference between 2 lines is $6.53 \mathrm{~mm}$. The $\mathrm{M} 1$ angle is 51.7 (Asterisk).

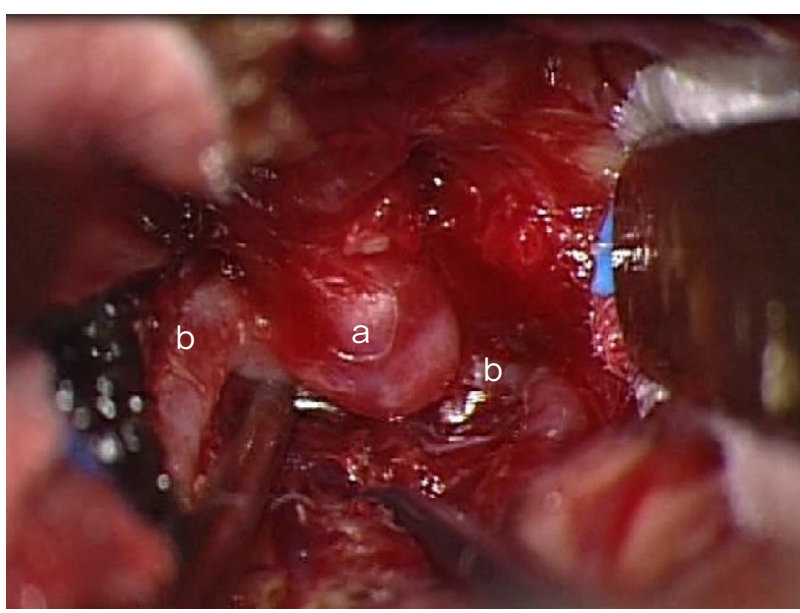

Fig. 4. An intra-operative view of the left MCA bifurcation aneurysm (a), M2 branches (b). It is difficult to identify M1 vessel.

proach, however, disadvantages are that early proximal control of M1 cannot be achieved and dissection cannot be made because of the complexity of sylvian veins in the periphery of the fissure. ${ }^{8)}$ So we combined 2 methods following surgeons' preference and experience.

Based on published studies about surgical approaches for sylvian fissure dissection, it is mandatory to evaluate the depth of sylvian fissure, the length and course of M1 and M2, and the projection and size of the MCA bifurcation aneurysm for neck clipping. ${ }^{4}$ In 1995, Christopher et al. reported that the distal to

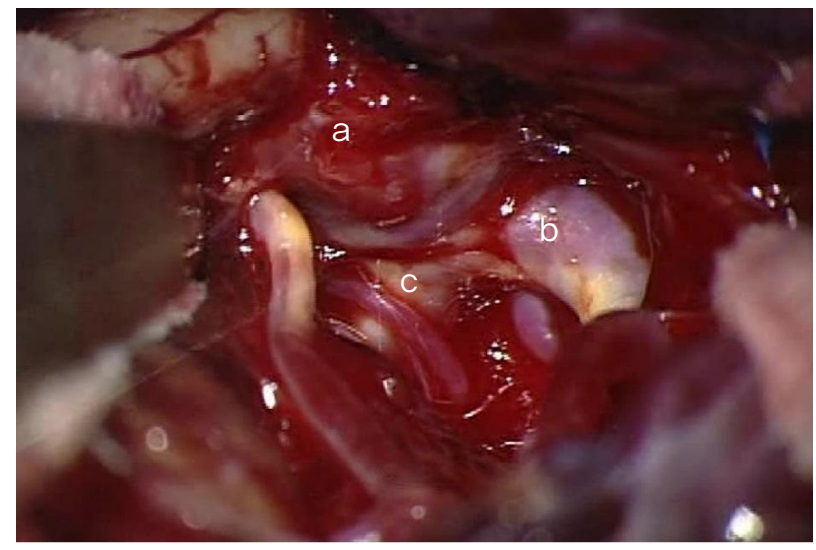

Fig. 6. An intraoperative view of the right MCA bifurcation aneurysm (a), M2 branches (b), M1 (c). It is easy to identify M1 vessel (c). 
proximal opening of the sylvian fissure could expose the lesion easily when there is a long M1. ${ }^{8)}$ Pritz et al. also reported that it would be the best treatment of choice in such cases. ${ }^{7)}$ Surgical approaches are also subject to the direction in which the aneurysm projection. That is, the sylvian fissure approach with proximal to distal exposure of the arterial anatomy would be ideal in cases of superiorly directed aneurysm. ${ }^{8)}$

Surgical approaches for sylvian fissure are determined based on the above factors and surgeons' preference and experience. We suggest that M1 exposure is a crucial procedure during aneurysm clipping, for which the direction of sylvian fissure dissection is an important step. It is important to identify whether there are parallel relationships between microscopic view angle and direction of M1 vessel or not. In other words, the lower the M1 angle and the smaller the M1 slope gap, the more parallel relationships between microscopic view angle and direction of M1 vessel, making it difficult to identify the M1 vessel through distal to proximal sylvian fissure dissection method. In those situations, we prefer the proximal to distal sylvian fissure dissection method. The high M1 angle and big M1 slope gap mean that the M1 exposure area is wide in the microscopic view so we can identify the M1 vessel easily, resulting in ease to perform M1 proximal control through distal to proximal sylvian fissure dissection method. Therefore, we measured angle relationships by using 3D-CTA. According to our study, the M1 slope gap was $1.00 \pm$ $0.42 \mathrm{~mm}$ and the mean of M1 angle was $9.36 \pm 3.73^{\circ}$, indicating difficult to identify M1 vessels through distal to proximal sylvian fissure dissection method. So if the M1 slope gap is less than $1.42 \mathrm{~mm}$ or the M1 angle is less than $13.09^{\circ}$, proximal to distal sylvian fissure dissection is preferable. For example, when the M1 slope gap was $1.31 \mathrm{~mm}$ and M1 angle was $10.2^{\circ}$ in preoperative 3D-CTA, we would better using a proximal to distal sylvian fissure dissection for ease of M1 exposure.
Patients with ruptured aneurysms are at increased risk of developing premature rupture. Therefore, proximal control of M1 is mandatory for surgical clipping. ${ }^{4)}$ Temporary clip placement is a valuable technique that is increasingly used for aneurysm clipping. Appropriate use of temporary artery occlusion is an important adjunct during aneurysm clipping surgery. ${ }^{2)}$ Temporary occlusion of the proximal arteries during surgery will reduce the pressure in the aneurysm fundus, improve the safety of aneurysm neck dissection, reduce the risk of developing premature rupture, and help reduce the increased morbidity and mortality associated with intraoperative aneurysm rupture. Particularly, in acute surgery, the anatomical situation around the aneurysm can be difficult due to bleeding and adhesions within the cistern. Moreover, when there is antero-superior projection of the MCA bifurcation aneurysms, the dome may adhere to the arachnoid membrane covering the sylvian fissure. This raises the risk of developing premature rupture during dissection and exposure. ${ }^{4}$ Otherwise, it would also be necessary to puncture and empty the aneurysm, essential for completely separating the efferent branches and stimulating them. With loss of tension in the aneurysm, definitive clip placement can be achieved without damaging or tightening the vessels. ${ }^{110) 12}$

To our knowledge, based on previous literature review, there are no objective preoperative criteria based on a 3D-CTA for determining the direction of sylvian fissure dissection for easy surgical dissection trajectory for proximal control of M1. This suggests that surgical approaches for the direction of sylvian fissure dissection should be determined based on surgeons' preference and experience. We measured the M1 angles and M1 slope gap on a 3D-CTA. This may lead to a safer operation by making proximal control easier in the event of premature rupture. Therefore, we used a preoperative 3D-CTA to identify the LS line, ICA bifurcation, MCA bifurcation and M1 angles. Thus, we could decide on the direction of sylvian fis- 
sure dissection for the proximal control of $\mathrm{M} 1$ in cases of MCA bifurcation aneurysm through 3D-CTA.

In conclusion, proximal control of M1 using a temporary clip is a key procedure during aneurysmal neck clipping for preventing premature rupture of aneurysm. Our results indicate that the M1 angles and M1 slope gap on a 3D-CTA are significant in estimating the M1 traveling direction.

There are several limitations of this study. First, we classified our clinical series of patients into the groups depending on the technical level of ease of M1 exposure solely based on our subjective judgment. Second, we did not consider several factors for easy surgical dissection trajectory for proximal control of M1. For example, the length of M1 influences direction of the sylvian fissure dissection. If M1 is short, it is too difficult to dissect distal to proximal direction due to aneurysm's deep location. In addition, if the aneurysm is large, regardless of the direction of M1, M1 proximal control is difficult. Also, the M1 can be covered by a large aneurysm. Therefore, these factors should be considered. Finally, further prospective studies are necessary. Because this study was a retrospective study of a small series, clinical application for large series is required.

\section{CONCLUSION}

The direction of M1 vessel is an important factor for M1 exposure during MCA bifurcation aneurysm surgery, and the direction of M1 vessel depends on the M1 angles and the M1 slope gap. They should be confirmed on a 3D-CTA preoperatively. We suggest that our results are useful in identification of M1 vessel for proximal control during surgical clipping, especially when the possibility of premature rupture is high in sylvian fissure dissection. So if the M1 slope gap is less than $1.42 \mathrm{~mm}$ or the M1 angle is less than $13.09^{\circ}$, proximal to distal sylvian fissure dissection is preferable to distal to proximal sylvian fissure dissection for M1 exposure.

\section{REFERENCES}

1. Ahn JS, Kwun BD. [Pterional, trans-sylvian approach for aneurysm surgery]. Korean J Cerebrovasc Disease. 2000 Sep;2(2):149-53. Korean.

2. Dashti R, Hernesniemi J, Niemela M, Rinne J, Porras M, Lehecka $M$, et al. Microneurosurgical management of middle cerebral artery bifurcation aneurysms. Surg Neurol. 2007 May;67(5):441-56.

3. Fakhry M. Tuberculum sellae meningiomas. Bull Alex Fac Med 2006;42(1).

4. Huh SK. [Microsurgical anatomy of the middle cerebral artery]. J Korean Neurosurg Soc. 1998 Dec;27(12):1769-73. Korean.

5. Joo JY. [Surgical management of middle cerebral artery aneurysms]. Korean J Cerebrovasc Surg. 2003 Sep;5(2): 117-22. Korean.

6. Kumar MV, Karagiozov KL, Chen L, Imizu S, Yoneda $\mathrm{M}$, Watabe $\mathrm{T}$, et al. A classification of unruptured middle cerebral artery bifurcation aneurysms that can help in choice of clipping technique. Minim Invasive Neurosurg. 2007 Jun;50(3):132-9.

7. Lavine SD, Masri LS, Levy ML, Giannotta SL. Temporary occlusion of the middle cerebral artery in intracranial aneurysm surgery: Time limitation and advantage of brain protection. J Neurosurg. 1997 Dec;87(6):817-24.

8. Ogilvy CS, Crowell RM, Heros RC. Surgical management of middle cerebral artery aneurysms: Experience with transsylvian and superior temporal gyrus approaches. Surg Neurol. 1995 Jan;43(1):15-22; discussion 22-4.

9. Ogilvy CS, Carter BS, Kaplan S, Rich C, Crowell RM. Temporary vessel occlusion for aneurysm surgery: Risk factors for stroke in patients protected by induced hypothermia and hypertension and intravenous mannitol administration. J Neurosurg. 1996 May;84(5):785-91.

10. Paulo MS, Edgardo S, Fernando M, Pablo P, Alejandro $\mathrm{T}$, Veronica V. Aneurysms of the middle cerebral artery proximal segment $(\mathrm{m} 1) \cdot$ Anatomical and therapeutic considerations - Revision of a series. Analysis of a series of the pre bifurcation segment aneurysms. Asian J Neurosurg. 2010 Jul;5(2):57-63.

11. Pritz MB, Chandler WF. The transsylvian approach to middle cerebral artery bifurcation/trifurcation aneurysms. Surg Neurol. 1994 Mar;41(3):217-9; discussion 219-20.

12. Smrcka M, Smrcka V. The benefit of a temporary vessel occlusion in aneurysm surgery. Bratisl Lek Listy. 2002; 103(12):473-6. 